\title{
DIELECTRIC RELAXATIONS IN ULTRA-THIN FILMS OF PMMA: ASSESSING THE LENGTH SCALE OF COOPERATIVITY IN THE DYNAMIC GLASS TRANSITION
}

\author{
$\underline{\text { M. Wübbenhorst }}{ }^{1}$, C.A. Murray ${ }^{2}$, J.A. Forrest ${ }^{3}$, J.R. Dutcher ${ }^{2}$ \\ ${ }^{1}$ Delft University of Technology, The Netherlands. \\ ${ }^{2}$ University of Guelph, Department of Physics, Canada. \\ ${ }^{3}$ University of Waterloo, Department of Physics, Canada.
}

\begin{abstract}
The local and cooperative dynamics of supported ultra-thin films ( $L=4-170 \mathrm{~nm}$ ) of poly(methyl methacrylate) was investigated by dielectric spectroscopy at frequencies from $0.1 \mathrm{~Hz}$ to $10^{6} \mathrm{~Hz}$ and temperatures from $250-$ $423 \mathrm{~K}$. To assess the effect of stereoregularity, three tacticities ( $i, a$ and $s$-PMMA) of similar molecular weight $M_{n}=120-140 \times 10^{3} \mathrm{~g} \mathrm{~mol}^{-1}$ were studied. A second $M_{n}$ value for $s$-PMMA was also studied. For i-PMMA, a decrease of the glass transition temperature $T_{\mathrm{g}}$ by up to $10 \mathrm{~K}$ was observed for a film thickness $L<25 \mathrm{~nm}$ (which is comparable to $R_{E E}$ ), indicated by a down-shift of the peak temperature $T_{\alpha}$ in the loss $\varepsilon^{\prime \prime}(T)$ at low and high frequencies and by a shift of the maximum in the apparent activation energy $E_{a}(T)$ of the $\alpha$-process. Changes in the relaxation strength and the peak shape are apparent for $L \leq 50 \mathrm{~nm}$. In addition, the $\beta$-relaxation probing the local mobility was investigated for $i$-, $a$ - and $s$ - PMMA. Interestingly, all polymers revealed a systematic speedup in the local dynamics for $L<25 \mathrm{~nm}$ which is accompanied by systematic changes in the relaxation strength. For i-PMMA, a clear relation between the thickness dependence of $\tau_{\beta}$ and $\tau_{\alpha}$ was found indicating that thickness dependences in the cooperative dynamics are predominantly induced by changes in the local mobility. These findings are consistent with the idea of confinement induced changes in the orientation and conformation statistics for thicknesses below $R_{\mathrm{EE}}$.
\end{abstract}

\section{Introduction}

The idea of cooperatively-rearranging regions (CRR), as first proposed by Adam and Gibbs, has proved to be a useful concept in trying to understand the glass transition. An attractive way to prove the existence of a characteristic length scale $\xi$ of the glass transition dynamics is to study glassforming materials confined to geometries with size of the order of $\xi$. Examples of suitable confined geometries include nanoporous glasses [1], porous membranes [2], ultra-thin polymer films [3, 4] or channel structures of zeolite host systems $[5,6]$.

Ultra-thin polymer films are a particularly simple system for the study of size-effects on the glass transition and have the advantage that the interfacial interaction is readily quantified. Since polymers enable the formation of stable freely-standing films [4] or films on substrates [3], ultra-thin polymer films have been studied increasingly since the pioneering work of Keddie, Jones and Cory [7]. For freely-standing polymer films, spectacular glass transition reductions up to $70 \mathrm{~K}$ (for PS) were found which depend on the film thickness and, above a critical molecular weight, also on the end-to-end distance $R_{\mathrm{EE}}$ of the polymer [8]. In contrast, much lower $T_{\mathrm{g}}$ reductions or even an increase in $T_{\mathrm{g}}$ were observed for supported films that clearly depend on the nature of the substrate-polymer interactions. The various, sometimes contradicting experimental results, mainly based on ellipsometric or Brillouin light scattering (BLS) measurements, suggest that the $T_{\mathrm{g}}$ reductions found in most experiments are not

a

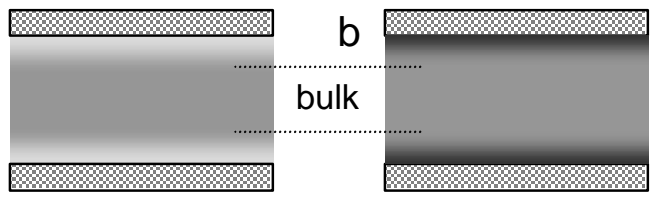

Fig. 1 Two-layer model for a sandwiched polymer films showing a) enhanced and b) reduced interfacial mobility.

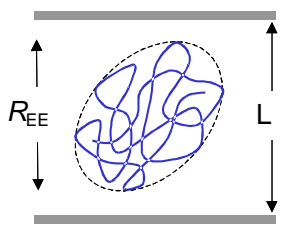

$L>R_{\mathrm{EE}}$

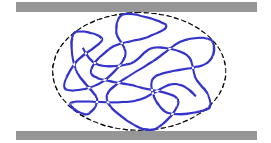

$L \sim R_{\mathrm{EE}}$

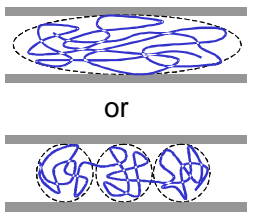

$L \ll R_{\mathrm{EE}}$
Fig. 2 Schematic configuration of a single chain in bulk and in confined geometry for $L<R_{\mathrm{EE}}$ causing orientation and deformation of the random coil. 
exclusively related to the size of the CCR. Instead, various mechanisms could be identified contributing to the (average) $T_{\mathrm{g}}$ : i) enhanced mobility close to a free (or weakly interacting) surface due to density anomalies ii) partial immobilization of polymer chains in the vicinity of polymer/substrate interfaces for strongly interacting (e.g. hydrogen bonded) polymers leading to a local increase in $T_{\mathrm{g}}$ (cf. Fig. 1b) and iii) enhancement of the mobility in the entire film for $L<R_{\mathrm{EE}}$ as a result of induced (in-plane) chain orientation and accompanying changes in the conformation statistics (see Fig. 2).

One of the consequences of the layer model is that the glass transition dynamics depends on the position within the film thickness which should result in a broadening of the $\alpha$-process upon decrease of the film thickness. Broadband dielectric relaxation spectroscopy (DRS) is perhaps one of the most suitable techniques to assess the dynamics of ultra-thin polar polymer films in a wide dynamic range and thus provides detailed spectral information about the dynamic glass transition. First DRS results on ultra-thin polymer films have been reported by Fukao [9], who studied dielectric relaxation properties of PVAc and atactic PMMA films as thin as $\sim 10 \mathrm{~nm}$, which were sandwiched between aluminium electrodes. Another DRS study on ultra-thin films was recently published by Hartmann et al. [10] who examined thin films of isotactic PMMA in the thickness range down to $18 \mathrm{~nm}$.

As extension to the few previous studies, we have studied for the first time both the local and cooperative dynamics of stereoregular PMMA as a function of tacticity and film thickness. In additon we were able to push the lower thickness limit of samples for the DRS studies down to about $4 \mathrm{~nm}$.

\section{Experimental}

Ultra-thin polymer films were prepared by spin-coating on cleaned glass slides onto which an $\sim 40 \mathrm{~nm}$ thick layer of $\mathrm{Al}$ had been evaporated. By varying the concentration of the polymer solution, the viscosity (molecular weight dependent) and the spin speed, homogeneous films in the thickness range from a few nanometer to about $200 \mathrm{~nm}$ were obtained. We used 3 types of PMMA with different tacticity having a common value of molecular weight of about $M_{\mathrm{w}} \sim 140 \times 10^{3} \mathrm{~g} / \mathrm{mol}$, as well as an additional sample of syndiotactic PMMA with a molecular weight of $880 \times 10^{3} \mathrm{~g} / \mathrm{mol}$. Following the deposition and annealing of the PMMA films, Al was gently evaporated onto the PMMA to form a patterned top electrode, yielding a sample consisting of 5 separate capacitors, providing redundancy in case of short circuits between the top and bottom electrodes. Surprisingly, the failure rate was very low for thicknesses greater than $20 \mathrm{~nm}$, and sufficiently low to allow dielectric measurements on samples as thin as $4 \mathrm{~nm}$.

DRS experiments in the frequency range from $10^{-1}$ to $10^{6} \mathrm{~Hz}$ were performed with a custom-made dielectric spectrometer using an RLC-meter HP4284A, a Schlumberger 1260 and an high-impedance dielectric interface. The sample temperature was controlled by a Novocontrol Quatro Cryosystem.

\section{Results and Discussion}

Before discussing thickness effects on the relaxation behavior, we will first address the bulk dynamics of PMMA as a function of tacticity. For this purpose, relatively thick samples ( $\left.L \gg R_{\mathrm{EE}}\right)$ of isotactic,

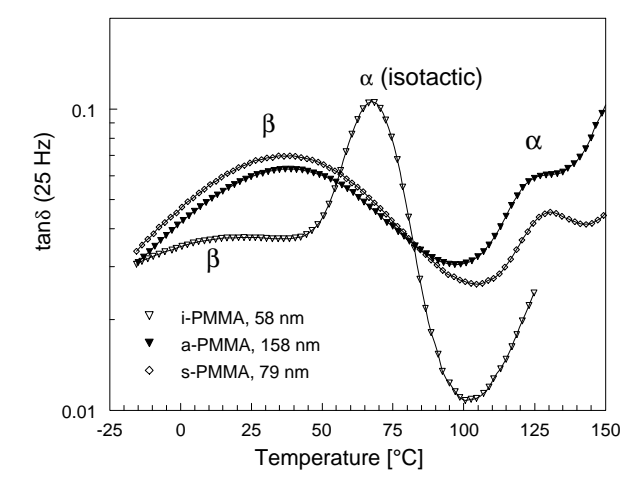

Fig. 3 Loss tangent at $f=25 \mathrm{~Hz}$ of i-, s- and aPMMA as function of temperature.

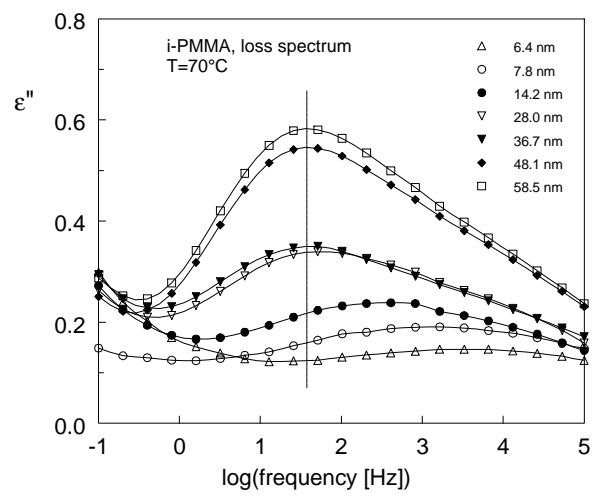

Fig. 4 The $\alpha$-relaxation peak of i-PMMA at $70^{\circ} \mathrm{C}$ 
atactic and syndiotactic PMMA were examined, which are expected to exhibit bulk-like dynamics. Fig. 3 shows the dielectric loss tangent at $f=25 \mathrm{~Hz}$ as a function of the temperature. All three polymers have a similar molecular weight $\left(M_{\mathrm{w}} \sim 140 \times 10^{3} \mathrm{~g} / \mathrm{mol}\right)$ and the loss curves reveal the two prominent relaxation processes of PMMA: the high-temperature $\alpha$-process associated with the dynamic glass transition, and the broad $\beta$-relaxation that was attributed to slightly cooperative, local motions involving the side-groups and the nearest part of the backbone in the glassy state [11].

We will now discuss the effect of decreasing the film thickness on the $\alpha$-relaxation peak. Since the $\alpha$ process is strongest for i-PMMA, we will focus on a quantitative analysis of the $\alpha$-process of isotactic PMMA. Fig. 4 shows the dielectric loss spectra at $70^{\circ} \mathrm{C}$ for 7 different film thicknesses from $60 \mathrm{~nm}$ down to about $6 \mathrm{~nm}$. Below about $50 \mathrm{~nm}$, the spectra start to deviate from the "bulk" spectrum by a reduction in the relaxation strength and a broadening of the $\alpha$-peak. Below a further critical thickness $(\sim 28 \mathrm{~nm})$, additionally a shift in the alpha-peak towards higher frequencies becomes visible. This upshift of the relaxation rate in the frequency domain is equivalent to a down-shift of the loss peak in the temperature representation according to Fig. 5, which displays the shift of the loss peaks with decreasing thickness for two distinct frequencies.

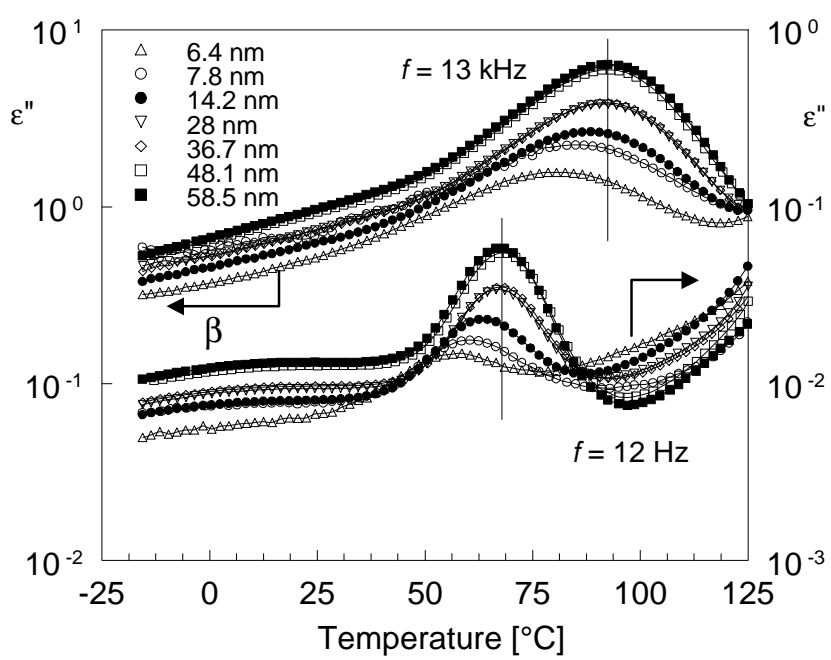

Fig. 5 Loss of i-PMMA at $f=6.4$ and $f=13 \mathrm{kHz}$ for different thicknesses as a function of the temperature. For more clarity the two series were shifted vertically with respect to each other.

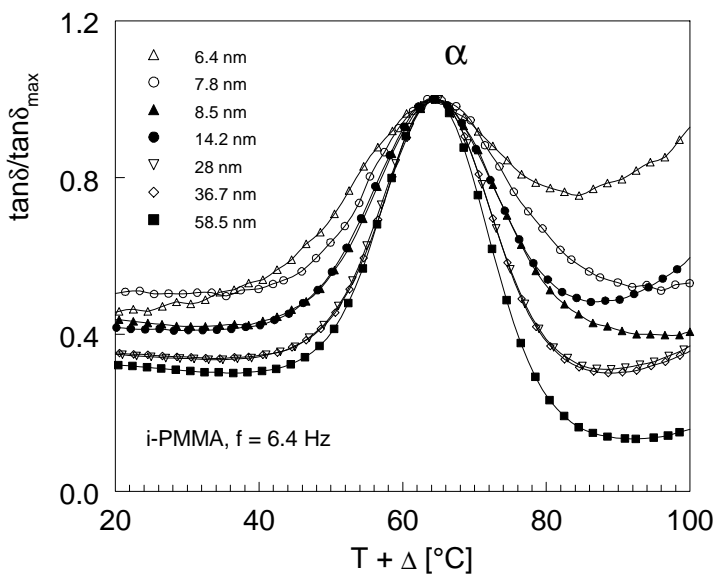

Fig. 6 Loss tangent of i-PMMA at $f=6.4 \mathrm{~Hz}$ shifted to the peak position $T_{\max }$ and normalized to the peak maximum $\tan \delta_{\max }$ of the "bulk" sample $(L=58 \mathrm{~nm})$.

The effect of peak broadening becomes clearer visible in Fig. 6, showing horizontally shifted and vertically normalized loss curves for various thicknesses. Upon thickness reduction, the $\alpha$-peak broadens increasingly whereby both the low temperature and high temperature slopes become flatter. Such a broadening of the $\alpha$-relaxation can generally be attributed to either changes in the intrinsic spectral shape of the $\alpha$-process (e.g. due to changes in the intermolecular cooperativity) or to a spatial distribution of regions having a local non-broadened dynamics. However, the symmetric change in the peak shape of the $\alpha$-process of i-PMMA strongly points to the existence of a distribution in relaxation times according to the layer scenario depicted in Fig. 1.

Let's now discuss in more detail the shift of the loss peak maxima with decreasing sample thickness. Surprisingly, the shifts in the loss peaks given in Fig. 5 show the same trend for low and high frequencies indicating that the $\alpha$-relaxation dynamics speeds-up as a whole (cf. also Fig. 7). This finding is in contradiction to recent results by Hartmann et al. [10] who, already for films in the order of $100 \mathrm{~nm}$, noticed a change in the relaxation time dependence $\tau(T)$ at low frequencies while the high frequency dynamics remained almost unaffected by thickness reductions. To deduce values for the glass transition temperature $T_{\mathrm{g}}$ from the dielectric experiments we have applied the so-called activation energy fine-structure analysis [12] which yields the local, apparent activation energy $E_{\text {app }}(T)$ from the dielectric spectra $\varepsilon^{\prime}(f, T)$. It has been demonstrated earlier, that this technique allows to determine an 
operationally defined $T_{\mathrm{g}}$ from the maximum in $E_{\text {app }}(T)$ which is usually close to the calorimetric and dilatometric glass transition temperature [13]. The $E_{\text {app }}(T)$ curves for i-PMMA are given in Fig. 8. It should be noted that the highest $T_{\mathrm{g}}$ values determined in this way $\left(T_{\mathrm{g}}=328 \mathrm{~K}\right)$ are fairly close to those reported in [10] $\left(T_{\mathrm{g}, \mathrm{DSC}}=331 \mathrm{~K}, T_{\mathrm{g}, \mathrm{DRS}}=327 \mathrm{~K}\right)$.

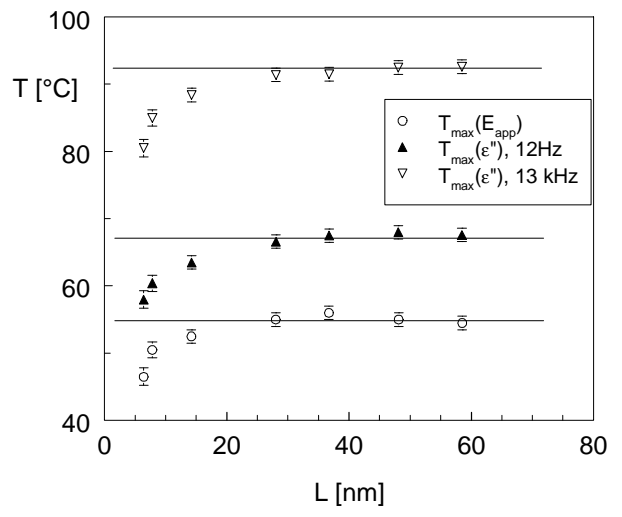

Fig. 7 Peak maximum temperatures and $T_{\mathrm{g}}$ obtained from the apparent activation energy for i-PMMA as function of the film thickness. The lines indicate the "bulk" limits.

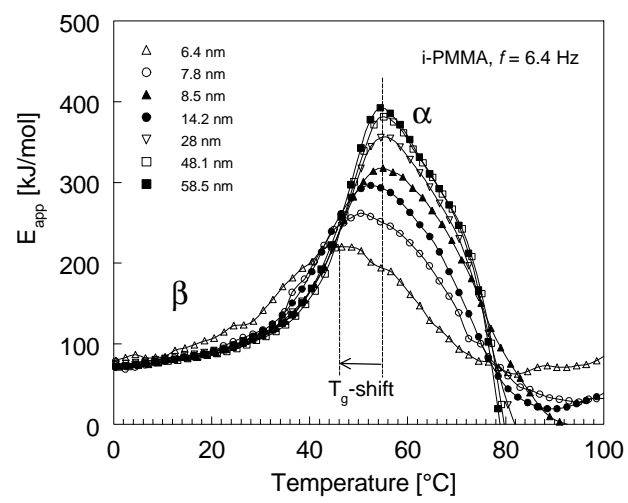

Fig. 8 Apparent activation energy vs. temperature isotactic PMMA sample of various thicknesses.

Although the $T_{\mathrm{g}}$ values obtained from $E_{\text {app }}(T)$ are generally lower than the loss peak temperatures determined from Fig. 5, all three dependences summarized in Fig. 7 show the same trend and maximum shift with respect to the bulk temperature of about $\sim 10 \mathrm{~K}$. Such a $T_{\mathrm{g}}$ depression is in good agreement with previous studies of PMMA films using other techniques. However, the critical thickness of about $30 \mathrm{~nm}$, at which substantial reductions in the glass transition temperature sets in is far different from the correspondent layer thickness of about 100nm as found by Hartmann et al. [10]. A substantially higher surface roughness of the Al-electrodes used in [10] caused by differences in the evaporation procedure, could provide a tentative explanation for these discrepancies, but needs further verification.

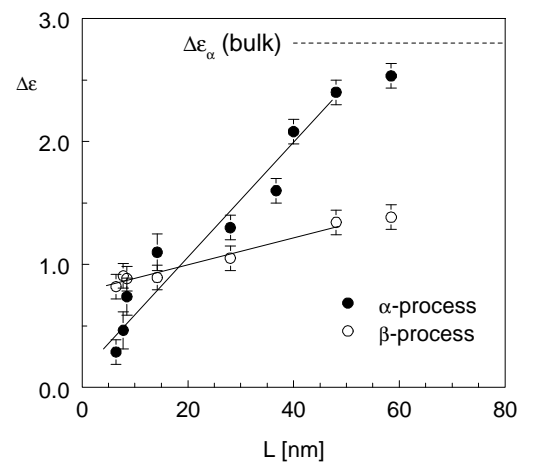

Fig. 9 Relaxation strength $\Delta \varepsilon(L)$ of iPMMA for the $\alpha$ - process (at $T=85^{\circ} \mathrm{C}$ ) and the $\beta$-process (at $T=30^{\circ} \mathrm{C}$ ). The bulk value for $\Delta \varepsilon_{\alpha}$ was taken from [10].

and Hartmann [10] did not lead to acceptable results since this model does not reproduce the particularly high changes at high film thicknesses.

\section{Thickness effects on the $\beta$-relaxation}

In contrast to the alpha-process, which characterizes cooperative motions involving a few repeating units, the $\beta$-relaxation reflects local conformational changes on
Among all changes of the $\alpha$-relaxation process with decreasing film thickness, the dependence of the relaxation strength $\Delta \varepsilon_{\alpha}(L)$ is probably the most striking one. An empirical fit of the $\Delta \varepsilon_{\alpha}(L)$ data with a linear function as shown in Fig. 9 suggests a critical film thickness in the order of $50 \mathrm{~nm}$. Interestingly, the strength of the $\beta$-process reveals a similar qualitative trend, although the absolute variation in $\Delta \varepsilon_{\beta}(L)$ is marginal compared to $\alpha$-process. Attempts to fit the $\Delta \varepsilon_{\alpha}(L)$ data to a layer model (cf. Fig. 1) as done by Fukao [9]

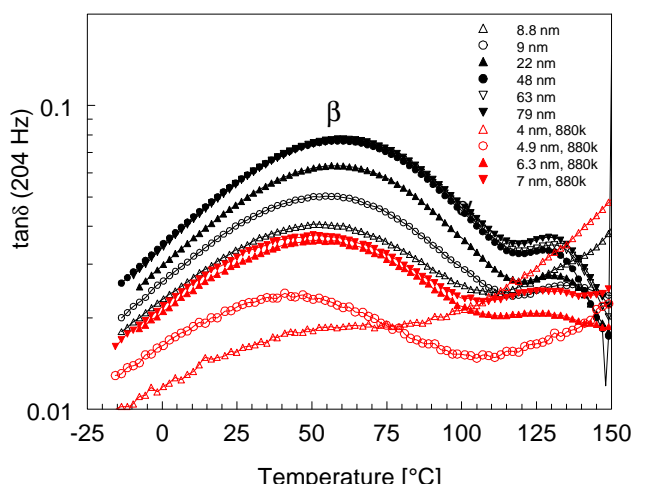

Fig. 10 Loss tangent vs. temperature at $f=$ $204 \mathrm{~Hz}$ for s-PMMA of different $M_{\mathrm{w}}$ and film thickness. 
the length scale of a few bond lengths, basically $<1 \mathrm{~nm}$. Because of its local nature, the $\beta$-process is not expected to be sensitive to finite size effects. In other words, the $\beta$-process should be homogeneous across the entire film thickness except at the polymer-metal interfaces where H-bonding and surface induced chain alignment might affect the local mobility.

Figure 10 displays the dielectric loss tangent at a fixed frequency as a function of temperature for syndiotactic PMMA of two different $M_{\mathrm{w}}$ 's. The black curves, corresponding to the lower $M_{\mathrm{w}}$ polymer, reveals a critical thickness dependence as already discussed in the previous section for the $\alpha$ - and $\beta$ relaxation of i-PMMA. While the $\beta$-peak is virtually unchanged as the film thickness is reduced from $78 \mathrm{~nm}$ down to $48 \mathrm{~nm}$, a sudden drop in the peak intensity and peak temperature starts, which is accompanied by a down-shift in the peak maximum temperature by up to $20 \mathrm{~K}$. For a quantitative analysis of the $\beta$-relaxation data we have fitted the spectra to the empirical Havriliak-Negami function

$$
\varepsilon^{\prime \prime}=\operatorname{Im}\left\{\Delta \varepsilon /\left(1+(i \omega \tau)^{a}\right)^{b}\right\}
$$

which is suitable to model asymmetrically broadened relaxation peaks by the shape parameters $a$ and $b$. The fit results for all three tacticities are summarized in Fig. 11 .
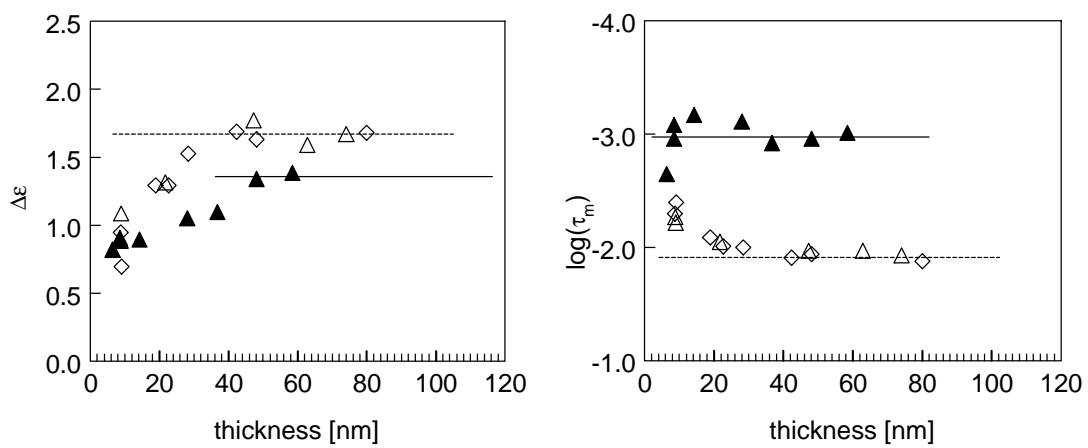

Fig. 11 Fit parameters for the $\beta$-process of $\mathrm{i}-$, aand S-PMMA $\left(M_{\mathrm{w}} \sim\right.$ $\left.140 \times 10^{3} \mathrm{~g} / \mathrm{mol}\right)$ at $T=$ $30^{\circ} \mathrm{C}$ as a function of film thickness. The dotted and solid lines are guide for the eyes and represent the limiting
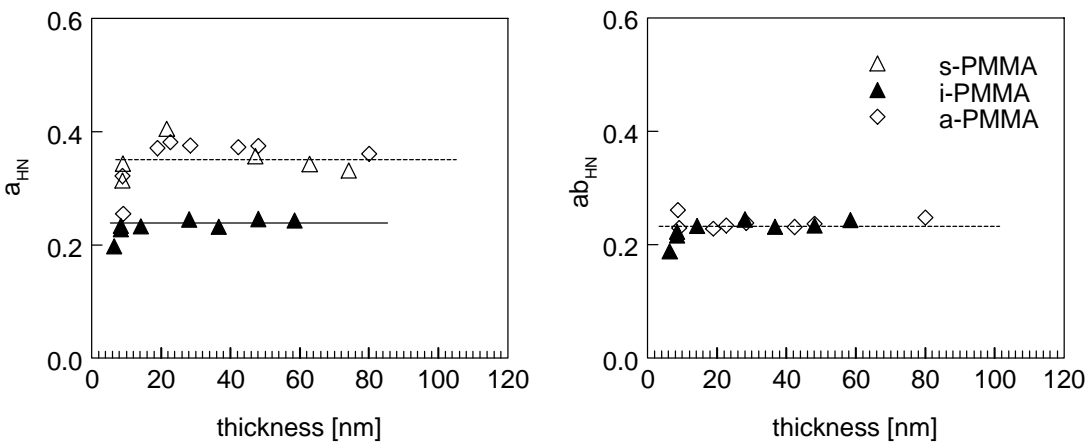
"bulk" values. Error bars have been omitted since they are in the order of the symbol size.

In analogy to the $\alpha$-process of i-PMMA, there is a significant increase in the relaxation rate $(1 / \tau)$ for $L$ $<30 \mathrm{~nm}$ as well as a decrease in the relaxation strength for $L$ around $40 \mathrm{~nm}$. Interestingly, the $\Delta \varepsilon_{\beta}(T)$ dependence is more pronounced for a-PMMA and s-PMMA compared to i-PMMA. On the other hand, thickness effects on the spectral distribution of the relaxation times, expressed by the shape parameters $a(L)$ and $b(L)$, are relatively small, indicating an almost homogeneous local dynamics across the film thickness. This behavior proves the local nature of the $\beta$-process of PMMA, making it insensitive to finite size effects. Since "dead" layers of substantial thickness or finite size effects can be ruled out, we think that chain confinement as illustrated in Fig. 2 is the prominent mechanism which causes the thickness dependence of the $\beta$-relaxation. When the film thickness becomes lower than the characteristic dimension of a single polymer chain, quantified by its end-to-end vector length $R_{\mathrm{EE}}$, the polymer random coil will be deformed to a "pancake"-shape and oriented which necessary results in a modification of the equilibrium conformational statistics (trans/gauche ratio) and orientation distribution of the polymer chains. Since this process results in more elongated chains (persistence length increases), the local molecular packing is improved which likely speeds-up the $\beta$-process. Further evidence for substantial alterations in the conformational statistics is provided by the 
relaxation strength $\Delta \varepsilon_{\beta}$ which, by virtue of sensing intra- and intermolecular dipole-dipole correlations, is susceptible to both the stereoregularity (tacticity) and the conformational statistics.

\section{Conclusions}

The dynamic glass transition and the local dynamics in supported ultrathin PMMA films were studied using dielectric spectroscopy. For three different tacticities, we have investigated the effect of film thickness in the range from 4 to $170 \mathrm{~nm}$ on both the $\alpha$ - and $\beta$-relaxation processes. Isotactic PMMA revealed reductions in the dielectric $T_{\mathrm{g}}$ by up to 10 degrees for films below $L=25 \mathrm{~nm}$, whereas a symmetric broadening of the alpha peak together with a reduction of the relaxation strength already sets in for samples below about 50nm. Surprisingly, the $\beta$-process shows a similar qualitative thickness dependence in its relaxation strength and its peak relaxation time. For $L \sim 40 \mathrm{~nm}$, a sharp decline in $\Delta \varepsilon_{\beta}$ becomes visible while an up-shift in the relaxation rate starts at $L<30 \mathrm{~nm}$.

The striking similarities between the $\alpha$ - and $\beta$-dynamics imply a common origin for the acceleration of the dynamics when reducing the film thickness below $L \sim R_{\mathrm{EE}}$. In this lower thickness range, the likely predominant mechanism is the overall change in the orientational and conformational statistics of the PMMA chains which results in a reduced relaxation strength and changes in intra- and intermolecular barriers for the $\beta$-process. For larger film thicknesses $(L \sim 25-50 \mathrm{~nm})$ changes in $\Delta \varepsilon_{\alpha}$ and $\Delta \varepsilon_{\beta}$ as well as broadening in the $\alpha$-peak might indicate the influence of a modified interface dynamics caused by surface interactions (reduced mobility) or surface induced chain alignment (enhanced mobility).

\section{References}

[1] P. Pissis, A. Kyritsis, G. Barut, R. Pelster, and G. Nimtz, "Glass transition in 2- and 3-dimensionally confined liquids," Journal of Non-Crystalline Solids, vol. 235, pp. 444-449, 1998.

[2] G. P. Crawford, D. K. Yang, S. Zumer, D. Finotello, and J. W. Doane, Phys. Rev. Lett., vol. 66, pp. 723, 1991.

[3] J. A. Forrest, K. DalnokiVeress, and J. R. Dutcher, "Interface and chain confinement effects on the glass transition temperature of thin polymer films," Physical Review E, vol. 56, pp. 5705-5716, 1997.

[4] J. A. Forrest, K. DalnokiVeress, J. R. Stevens, and J. R. Dutcher, "Effect of free surfaces on the glass transition temperature of thin polymer films," Physical Review Letters, vol. 77, pp. 2002-2005, 1996.

[5] A. Huwe, F. Kremer, P. Behrens, and W. Schwieger, Phys. Rev. Lett., vol. 82, pp. 2338, 1999.

[6] M. Wübbenhorst, G. J. Klap, J. C. Jansen, H. van Bekkum, and J. van Turnhout, "Glass transition of onedimensional molecular chains of $p \mathrm{NA}$ confined in $\mathrm{AlPO}_{4}-5$ nanopores revealed by dielectric spectroscopy," J. Chem. Phys., vol. 111, pp. 5637-5640, 1999.

[7] J. L. Keddie, R. A. L. Jones, and R. A. Cory, "Size-Dependent Depression of the Glass-Transition Temperature in Polymer-Films," Europhysics Letters, vol. 27, pp. 59-64, 1994.

[8] J. Mattsson, J. A. Forrest, and L. Borjesson, "Quantifying glass transition behavior in ultrathin freestanding polymer films," Physical Review E, vol. 62, pp. 5187-5200, 2000.

[9] K. Fukao, S. Uno, Y. Miyamoto, A. Hoshino, and H. Miyaji, "Dynamics of $\alpha$ - and $\beta$-processes in thin polymer films: poly(vinyl acetate) and poly(methyl methacrylate)," cond-mat, 2001.

[10] L. Hartmann, W. Gorbatschow, J. Hauwede, and F. Kremer, "Molecular Dynamics in Thin Films of Isotactic PMMA," European Physical Journal E, vol. submitted, 2001.

[11] K. Schmidt-Rohr, A. S. Kulik, H. W. Beckham, A. Ohlemacher, U. Pawelzik, C. Boeffel, and H. W. Spiess, "Molecular Nature of the $\beta$ Relaxation in Poly(methyl methacrylate) Investigated by Multidimensional NMR," Macromolecules, vol. 27, pp. 4733-4745, 1994.

[12] J. van Turnhout and M. Wübbenhorst, "Analysis of complex dielectric spectra. II. Evaluation of the activation energy landscape by differential sampling.," J. Non-Cryst. Solids, vol. 305, pp. 50-58, 2002.

[13] I. J. A. Mertens, M. Wübbenhorst, W. D. Oosterbaan, L. W. Jenneskens, and J. van Turnhout, "Novel Polymer Electrolytes Based on Amorphous Poly(ether-ester)s Containing 1,4,7-Trioxanonyl Main Chain Units. Ionic-Conductivity versus Polymer Chain Mobility," Macromolecules, vol. 32, pp. 3314-3324, 1999. 